\title{
SLIM FLOOR: MÉTODO DE DIMENSIONAMENTO E ESTUDO PARAMÉTRICO
}

\author{
Slim floor: Scaling method and study parametric
}

\author{
Vander Júnior de Mesquita Santos', Débora Machado de Lima², Felipe Isamu Harger Sakiyama ${ }^{3}$
}

Recebido em 23 de maio de 2016; recebido para revisão em 19 de agosto de 2016; aceito em 20 de setembro de 2016; disponivel on-line em 17 de novembro de 2016.

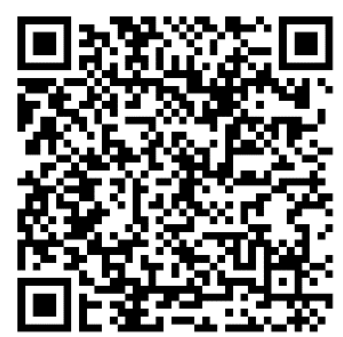

PALAVRAS CHAVE:

Slim floor;

Pisos mistos;

Vigas de aço;

Steel deck;

Dimensionamento.

\section{KEYWORDS:}

Slim floor;

Mixed floors;

Steel beams;

Steel deck;

Dimensioning.

RESUMO: Slim Floor é um sistema de pisos mistos aço-concreto de pequena elevação no qual a laje de concreto encontra-se embutida na altura da viga de aço, estando apoiada na mesa inferior do perfil. Este trabalho teve como objetivo estudar essa tipologia de estrutura, verificando a sua eficiência frente à conformação mista convencional de lajes e vigas, comumente conhecida como Steel Deck. Este artigo apresenta a metodologia de dimensionamento de um pavimento Slim Floor bem como os resultados de cálculo para uma estrutura Steel Deck, obtidos por meio do software VigaMix. Foram realizadas análises que envolveram parâmetros como o peso da viga, altura do piso, consumo de concreto e deformação. Dessa maneira, o sistema Slim Floor apresentou vantagens quanto aos aspectos construtivos, econômicos, estéticos e, principalmente em relação à altura total do pavimento. A maior contribuição deste estudo foi oferecer uma orientação para o dimensionamento de Slim Floor, visto que os critérios para a concepção deste tipo de estrutura ainda não se encontram totalmente consolidados nas normas atuais.

ABSTRACT: Slim floor system is a mixed steel-concrete floors small elevation in which the concrete slab is built on the height of the steel beam, being supported on the lower flange of the profile. This work aimed to study this type of structure, checking their front efficiency to conventional mixed forming slabs and beams, commonly known as Steel Deck. This paper presents the design methodology of a floor Slim Floor as well as the calculation results for Steel Deck structure obtained through VigaMix software. Analyses were performed involving parameters such as the weight of the beam, floor height, concrete consumption and deformation. In this way, Slim Floor system had advantages as the constructive, economic, aesthetic, and especially in relation to the total height of the floor. The major contribution of this study was to provide guidance for the design of Slim Floor, as the criteria for the design of this type of structure has not yet been fully consolidated in the current standards.

* Contato com os autores:

${ }^{1}$ e-mail: vanderjunior3m@hotmail.com (V.J.M. Santos)

Graduando em Engenharia Civil pela Universidade Federal dos Vales do Jequitinhonha e Murici.

${ }^{2}$ e-mail: deboramachadoli@hotmail.com (D. M. de Lima)

Graduando em Engenharia Civil pela Universidade Federal dos Vales do Jequitinhonha e Murici.

3e-mail: felipe.sakiyama@ufvjm.edu.br (F. I. H. Sakiyama)

Engenheiro Civil, Mestre, Professor da Universidade Federal dos Vales do Jequitinhonha e Murici. 


\section{INTRODUÇÃO}

Estruturas mistas são caracterizadas pela associação de dois ou mais materiais de forma a aproveitar as melhores qualidades dos mesmos, a fim de aumentar sua capacidade resistente e a eficiência do comportamento em serviço. As possibilidades de combinações de materiais são várias. O presente trabalho trata da interação entre laje de concreto com forma de aço incorporada e viga de aço, unidas por meio de conectores de cisalhamento que transferem esforços entre os materiais garantindo o comportamento misto.

Em pavimentos mistos de concreto e aço convencionais, a laje encontra-se apoiada na mesa superior do perfil da viga metálica, o que a caracteriza como sendo de altura elevada. Além disso, esse tipo de estrutura, dependendo da função da construção, pode necessitar por questões estéticas da utilização de forros suspensos. Essa solução implica no aumento do pé direito a ser construído, mantendo uma forma arquitetônica recomendável.

Com o desenvolvimento de estudos relacionados às lajes mistas, novas soluções estão sendo criadas. Este artigo apresenta uma tipologia denominada Slim Floor (piso misto de pequena altura).

Slim Floor é um sistema misto no qual a laje encontra-se apoiada na mesa inferior do perfil metálico, estando embutida na altura da viga. Para receber a laje, a mesa inferior do perfil apresenta maior largura do que a mesa superior. Desde meados do século XIX os princípios deste sistema eram utilizados e, a partir da década de 70, começaram a ser definidos na comunidade técnica internacional (PAES, 2003).

Os pisos mistos de pequena altura possuem vantagens relevantes em relação às estruturas convencionais. A camada de concreto que envolve a viga metálica, exceto a mesa inferior, aumenta a resistência da estrutura em situações de incêndio e à corrosão metálica, diminuindo ou tornando desnecessário o uso de aditivos de proteção. De acordo com Barros (2011), com o sistema misto Slim Floor é possível alcançar vãos de viga de $8 \mathrm{~m}$ e vãos de laje que variam de $10 \mathrm{~m}$ a
$12 \mathrm{~m}$, viabilizando a criação de espaços abertos com número reduzido de pilares.

Além de permitir a instalação de equipamentos técnicos (sistema elétrico e hidráulico, por exemplo) entre as nervuras da laje, o Slim Floor pode ser executado tão rápido quanto as lajes pré-fabricadas e lajes maciças. $O$ fato de a viga de aço estar integrada à altura total do pavimento diminui irregularidades no piso, reduzindo gastos com o acabamento e garante uma altura menor do que a de vigas mistas convencionais.

A última característica citada é o principal benefício do Slim Floor. Entretanto, conduz a uma importante análise: quanto menor a altura do sistema, maior a sua deformabilidade, o que exige as verificações dos Estados Limites de Serviço, em especial no controle de flechas e vibrações. Todavia, a exploração de amplas possibilidades construtivas do Slim Floor é uma alternativa bastante atrativa e que requer estudos mais aprofundados do comportamento estrutural destes sistemas (PAES, 2003).

\section{OBJETIVO}

O objetivo deste trabalho é contribuir para o avanço dos estudos sobre o sistema de estrutura mista Slim Floor, apresentando a metodologia de dimensionamento segundo normas técnicas internacionais e realizando uma análise paramétrica para comparar sua eficiência em relação a uma estrutura mista convencional comumente conhecida como Steel Deck.

\section{REVISÃO BIBLIOGRÁFICA}

O desenvolvimento de construções mistas de aço e concreto deve-se a um conceito básico: o aço resiste bem à tração e o concreto tem alta resistência à compressão. Dessa forma, têm por objetivo aproveitar as melhores qualidades de cada material, não somente a respeito da capacidade resistente, mas também do ponto de vista construtivo, funcional e estético, almejando minimizar as complicações intrínsecas que cada elemento possui (PAES, 2003).

Não há um consenso na literatura sobre a 
data exata que esse tipo de construção começou a ser utilizado. Através da observação dos trabalhos de De Nardin (2003), Griffis (1994) e Malite (1993), pode-se inferir o surgimento do sistema em meados do século XIX no Reino Unido e início do século XX nos Estados Unidos da América.

Segundo De Nardin et al. (2005), para que os dois materiais trabalhem concomitantemente na resistência aos esforços solicitantes, é preciso garantir o comportamento conjunto, isto é, os materiais devem se deformar como um único elemento, resultando uma configuração mista. Para isso, o fluxo de cisalhamento criado na interface entre aço e o concreto deve ser absorvido por algum tipo de conexão. Há diversas maneiras de possibilitar a aderência dos componentes, entre as quais pode-se citar a utilização de dispositivos mecânicos como conectores de cisalhamento, mossas e saliências.

Vale ressaltar que uma estrutura mista aço-concreto é composta por elementos mistos, sendo de relevância para a construção desta pesquisa as vigas e lajes. De acordo com Dos Reis (2012), o desempenho misto das vigas é assegurado pela conexão entre o concreto e o perfil de aço por meio de conectores de cisalhamento. Em geral os conectores são do tipo pino com cabeça ou em perfil $U$, os quais podem ser dispostos nas mesas superior e inferior, ou na alma. A interação pode ser estabelecida também por ranhuras presentes na superfície da mesa superior do perfil.

Em contrapartida, para as lajes mistas, a presença da chapa metálica deve ser capaz de transmitir o cisalhamento na aderência aço-concreto certificando o trabalho solidário dos dois materiais. Porém, a aderência entre a forma e o concreto não é suficiente, e dessa forma, a ligação é feita por meio de mossas ou por atrito gerado pelo confinamento do concreto em formas reentrantes (NARDIN et al., 2005).

\subsection{PISO MISTO DE PEQUENA ALTURA (SLIM FLOOR)}

A estrutura Slim Floor é configurada por vigas metálicas de altura reduzida e mesa inferior com maior largura do que a superior, de modo que a laje possa ser apoiada. Como o próprio nome diz, é um tipo de pavimento de espessura reduzida, no qual a viga encontra-se inserida na altura da laje de concreto. Essa característica é o principal diferencial em relação às estruturas mistas convencionais de aço e concreto.

Apesar de sua considerável utilização na Europa, o sistema Slim Floor ainda está em processo de estudo e desenvolvimento. Vale ressaltar a ausência de normas que assegurem todo o processo de dimensionamento e existência de poucos trabalhos técnicos publicados, o que dificulta o processo de consolidação dessa estrutura no âmbito internacional.

No Brasil, existe no mercado um sistema constituído por vigas metálicas e uma laje mista moldada in loco, no qual a chapa nervurada é apoiada no banzo inferior da viga e a camada de concreto alcança a altura do banzo superior (200 mm a $250 \mathrm{~mm}$ ). Alguns conectores são colocados horizontalmente, na alma, e verticalmente na mesa inferior da viga. Entretanto, apesar de econômico e atrativo, o sistema não tem comportamento misto e as vigas são calculadas como aço isolado (PAES, 2003).

\subsubsection{Classificação das seções mistas}

Segundo a norma EN 1994-1-1 (ECS, 2004), pode-se classificar as seções mistas de acordo com a análise global plástica, que pode ser utilizada quando os elementos que compõem a seção têm uma capacidade de rotação suficiente para permitir as redistribuições de momentos fletores.

Tal normativa indica ainda que essa classificação pode ser realizada como disposto na EN 1993-1-1 (ECS, 2010). Dessa maneira, são definidas quatro classes de seções transversais da seguinte forma:

- Classe 1: aquelas em que se pode formar uma rótula plástica, com a capacidade de rotação necessária para uma análise plástica, sem redução da sua resistência;

- Classe 2: aquelas que podem atingir o momento resistente plástico, mas cuja capacidade de rotação é limitada pelo escoamento do aço local;

- Classe 3: aquelas em que a tensão na 
fibra extrema comprimida, calculada com base numa distribuição elástica de tensões, pode atingir o valor da tensão de escoamento, mas em que o escoamento do aço local pode impedir que o momento resistente plástico seja atingido;

- Classe 4: aquelas em que ocorre o escoamento do aço local antes de se atingir a tensão de escoamento numa ou mais partes da seção transversal.

A classificação de uma seção transversal depende da esbeltez dos elementos comprimidos, do tipo de aço e do processo de fabricação da seção (laminada ou soldada) (PAES, 2003).

Os perfis de aço para as vigas Slim Floor, por recomendação, devem ser de Classe 1 (plásticas) ou Classe 2 (compactas). As seções de Classes 3 ou 4 limitam a resistência ao momento elástico resistente, o que é considerado antieconômico no atual contexto do dimensionamento de estruturas de aço (PAES, 2003).

\subsubsection{Tipos de vigas Slim Floor em perfil I ou H}

A partir do momento em que os princípios da Slim floor começaram a ser definidos pela comunidade técnica internacional, várias alternativas construtivas com tipos de perfis metálicos distintos foram desenvolvidas. Atualmente, os perfis comumente utilizados são: Integrated Floor Beam (IFB), Asymmetric Slimflor Beam (ASB) e Slimflor Fabricated Beam (SFB).

$A$ seção IFB é constituída por uma chapa de aço soldada na metade da alma de um perfil laminado I ou $\mathrm{H}$, formando uma seção assimétrica. Existem dois tipos de viga IFB, apresentados na Figura 1. O tipo A consiste de um perfil ( ou $\mathrm{H})$ cortado em formato de $T$ no qual é soldada uma mesa mais larga. O tipo $\mathrm{B}$, ao contrário, ocorre quando uma mesa mais espessa é soldada ao perfil cortado em formato de T invertido (BARROS, 2011). Para esta seção a interação entre o aço e o concreto é feita por conectores de cisalhamento.

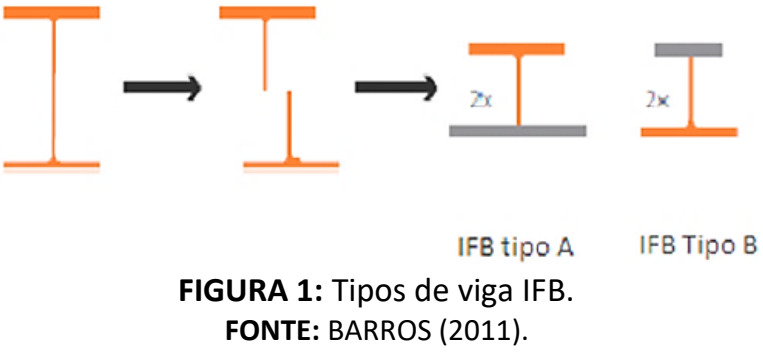

A seção ASB trata de um perfil laminado assimétrico, na qual a mesa inferior possui uma largura maior do que a da mesa superior (Figura 2). A superfície da mesa superior possui ranhuras introduzidas durante o processo de laminação com função de transferir esforços de cisalhamento por aderência (PAES, 2003).

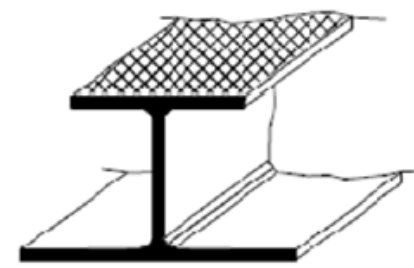

FIGURA 2: Asymmetric Slimflor Beam (ASB). FONTE: BARROS (2011).

O perfil SFB (Figura 3) foi desenvolvido no início dos anos 90 do século $\mathrm{XX}$, quando o desenvolvimento do sistema Slim Floor tornou-se de grande interesse para o Reino Unido. Na busca por possiblidades inovadoras, em 1992 a Britsh Steel (atual Tata Steel) e o Steel Construction Institute (SCl) uniram-se para a criação dessa seção caracterizada por um perfil I simétrico com uma placa de maior largura soldada à mesa inferior (BARROS, 2011). Atualmente, existem outras empresas produzindo esse tipo de perfil, como por exemplo o Grupo ArcelorMittal.

A transferência de esforços entre os materiais de um sistema Slim Floor com viga SFB é realizada, geralmente, por conectores de cisalhamento tipo pino com cabeça.

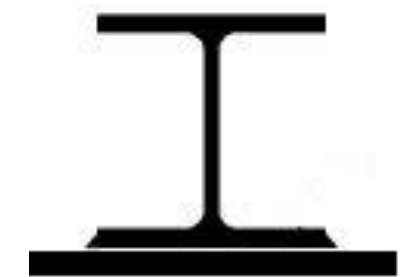

FIGURA 3: Slimflor Fabricated Beam (SFB). FONTE: PAES (2003). 


\subsubsection{Grau de interação entre os materiais}

Quando a forma da construção consta de um sistema de vigas mistas, o momento fletor resistente é determinado em função do grau de conexão entre o aço e o concreto, que pode ser total ou parcial (PAES, 2003).

Uma viga mista de Slim Floor tem conexão total quando o número de conectores é, no mínimo, igual ao que se requer para assegurar a mobilização do momento plástico resistente da seção mista. Isto é, a conexão total permite alcançar o máximo momento fletor resistente de uma seção transversal (BARROS, 2011).

A conexão parcial ocorre quando o número de conectores utilizados não é suficiente para transmitir a capacidade total das seções de concreto e aço (PAES, 2003). A utilização da conexão parcial diminui o momento plástico resistente, dessa forma é importante a definição do comportamento da diminuição da resistência e de que forma isso influencia no comportamento em serviço.

\section{METOTOLOGIA}

\subsection{CONSIDERAÇÕES PRELIMINARES}

O dimensionamento de vigas Slim Floor compreende as verificações no Estado Limite Último (ELU) e no Estado Limite de Serviço (ELS), independente da tipologia construtiva. Os ELU compreendem a verificação da resistência da seção da viga frente aos diferentes tipos de esforços e suas respectivas interações. Em contrapartida, os ELS abordam os efeitos das flechas e vibrações (PAES, 2003).

Assim como ocorre em estruturas mistas em geral, o dimensionamento de vigas Slim Floor requer a identificação e a consideração das distintas situações de cálculo para as combinações de ações normais e de construção. Este trabalho limita-se a expor para o ELU os cálculos referentes à etapa de utilização, considerando o trabalho misto da estrutura, e para o ELS, os cálculos referentes às deformações.

Para o dimensionamento e a obtenção dos resultados foram utilizados: os códigos europeus EN 1993-1-1 (ECS, 2010), EN 1994-1-1 (ECS, 2004); as normatizações brasileiras NBR 8800 (ABNT, 2008), NBR 6120 (ABNT, 1980); o programa computacional VigaMix desenvolvido pela Universidade Federal de Viçosa; e planilhas eletrônicas.

O dimensionamento do Slim Floor requer a definição de uma disposição construtiva para o sistema, ou seja, os tipos de viga e laje que serão utilizadas nas análises.

\subsubsection{Tipo de viga}

Empregou-se a Viga Slim Floor do tipo Slimflor Fabricated Beam (SFB), produzida pelo Grupo ArcelorMittal.

\subsubsection{Tipo de laje}

Utilizou-se a laje mista com concreto moldado in loco, composta por uma forma de aço trapezoidal nervurada de grande altura tipo PMF Comflor 210 (CF 210), produzida pelo Grupo Tata Steel, com as dimensões apresentadas na Figura 4.

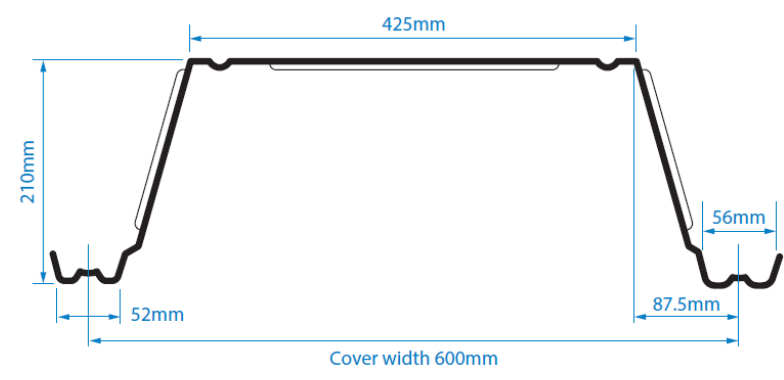

FIGURA 4: Dimensões chapa PMF ComFlor 210 (CF 210). FONTE: TATA STEEL (2013).

As propriedades da seção da chapa (por metro de largura) e o consumo de concreto (em metros cúbicos por metro quadrado), de acordo com a altura total do piso, foram obtidos através do catálogo - Comflor composite floor deck brochure disponibilizado por Tata Steel (2013).

\subsection{FORMULAÇÕES NO ELU}

\subsubsection{Seções mistas}

Para a escolha do perfil de viga que irá compor o sistema Slim Floor, deve-se verificar se a seção transversal pode ser classificada como classe 1 ou 2.

\subsubsection{Resistência ao momento fletor}

Neste trabalho utilizou-se a interação total por meio de conectores de cisalhamento do tipo 
pino com cabeça. Segundo Paes (2003), o momento fletor resistente de uma viga mista Slim Floor com conectores de cisalhamento, considerando interação total e com uma seção mista eficaz de classe 1 ou 2, pode ser determinado empregando a teoria plástica apresentada na EN 1994-1-1 (ECS, 2004), de maneira semelhante ao que se faz para as vigas mistas convencionais.

Para a determinação do momento fletor plástico resistente de cálculo $\left(\mathrm{M}_{\mathrm{pl}, \mathrm{Rd}}\right)$ é necessário conhecer a posição da linha neutra plástica $\left(\mathrm{y}_{\mathrm{p}}\right)$, medida a partir do topo de concreto da laje. Segundo Barros (2011), uma estrutura Slim Floor composta por perfil SFB combinado com laje com forma de aço incorporada, para $D_{d}<h_{\text {eff }}-t_{f}$, tem cinco possibilidades para $y_{p}$ e consequentemente cinco valores para $\mathrm{M}_{\mathrm{pl}, \mathrm{Rd}}$.

A Equação 1 indica $y_{p}$ na mesa superior do perfil metálico. Portanto, o $\mathrm{M}_{\mathrm{pl}, \mathrm{Rd}}$ é definido, conforme Paes (2003), a partir da soma dos momentos resistentes de cada resultante de tensão em relação a $y_{p}$, de acordo com a Equação 2. As Equações 1 e 2 foram adaptadas dos estudos de BARROS (2011).

Por último, a verificação da resistência à flexão é realizada de acordo com a Equação 3.

Um parâmetro importante que deve ser definido antes das formulações é o valor da largura efetiva da laje de concreto $\left(b_{\text {eff }}\right)$ que, para uma interação por meio de conectores, é tomada como um quarto do vão da viga.

$y_{p}=\left(D_{s}+D_{d}-h_{\text {eff }}\right)+\left(\frac{\frac{f_{y}}{\gamma_{a 1}} A_{a}-\frac{0,85 f_{c k}}{\gamma_{c}} b_{\text {eff }}\left(D_{s}+D_{d}-h_{\text {eff }}\right)}{2 \frac{f_{y}}{\gamma_{a 1}} b+\frac{0,85 f_{c k}}{\gamma_{c}} b_{\text {eff }}}\right)$

$M_{p l, R d}=\frac{0,85 f_{c k}}{\gamma_{c}} b_{\text {eff }} D_{s}\left(y_{p}-\frac{D_{s}}{2}\right)+$

$\left(\left(y_{p}-D_{s}-D_{d}+h_{e f f}\right)^{2}\right) \frac{f_{y} b}{2 \gamma_{a 1}}+$

$\left(\left(D_{s}+D_{d}-h_{e f f}+t_{f}-y_{p}\right)^{2}\right) \frac{f_{y} b}{2 \gamma_{a 1}}+$

$\left(h_{\text {eff- }} t_{f}\right) t_{w}\left(D_{s}+D_{d}-\frac{h_{\text {eff }}}{2}-y_{p}\right) \frac{f_{y}}{\gamma_{a 1}}+$

$$
\begin{aligned}
& t_{f} b\left(D_{s}+D_{d}-y_{p}-\frac{t_{f}}{2}\right) \frac{f_{y}}{\gamma_{a 1}}+ \\
& t_{p} b_{p}\left(D_{s}+D_{d}-y_{p}+\frac{t_{p}}{2}\right) \frac{f_{y}}{\gamma_{a 1}} \\
& M_{S d} \leq M_{p l, R d}
\end{aligned}
$$

\section{Em que:}

$\mathbf{y}_{\mathbf{p}}=$ Posição da linha neutra plástica $(\mathrm{m})$;

$\mathbf{M}_{\mathbf{p l}, \mathbf{R d}}=$ Momento fletor plástico resistente de cálculo (kN.m);

$\mathbf{M}_{\mathrm{Sd}}=$ Momento fletor solicitante de cálculo (kN.m); $\mathbf{D}_{\mathbf{s}}=$ Altura de concreto colocado in loco acima da chapa nervurada $(\mathrm{m})$;

$\mathbf{D}_{\mathbf{d}}=$ Altura da chapa nervurada $(\mathrm{m})$;

$\mathbf{b}_{\text {eff }}=$ Largura efetiva da laje de concreto $(\mathrm{m})$;

$\mathbf{h}_{\text {eff }}=$ Altura total do perfil sem a chapa adicional (m);

$A_{a}=$ Área total de aço $\left(\mathrm{m}^{2}\right)$;

$\mathbf{f}_{\mathbf{y}}=$ Resistência ao escoamento do aço (kPa);

$\mathbf{f}_{\mathbf{c k}}=$ Resistência característica do concreto à compressão ( $\mathrm{kPa})$;

$\mathbf{V}_{\mathbf{c}}=$ Coeficiente de ponderação da resistência do concreto, igual a 1,4 ;

$\boldsymbol{V}_{\mathrm{a} 1}=$ Coeficiente de ponderação da resistência do aço, igual a 1,1 ;

$\mathbf{b}=$ Largura da mesa do perfil $(\mathrm{m})$;

$\mathbf{t}_{\mathbf{f}}=$ Espessura da mesa do perfil $(\mathrm{m})$;

$\mathbf{t}_{\mathbf{w}}=$ Espessura da alma do perfil $(\mathrm{m})$;

$\mathbf{b}_{\mathbf{p}}=$ Largura da mesa da chapa adicional (m);

$\mathbf{t}_{\mathbf{p}}=$ Espessura da chapa adicional $(\mathrm{m})$.

\subsubsection{Resistência ao esforço cortante}

Segundo Paes (2003), em geral, considerase que somente a seção de aço resiste ao esforço cortante solicitante $\left(\mathrm{V}_{\mathrm{Sd}}\right)$, desprezando a contribuição do concreto.

A força cortante resistente de cálculo pode ser estimada baseada na EN 1993-1-1 (ECS, 2010), mediante à Equação 4.

$$
V_{R d}=\frac{A_{v}\left(\frac{f_{y}}{\sqrt{3}}\right)}{\gamma_{a 1}}
$$

Em que:

$\mathbf{V}_{\mathbf{R d}}=$ Força cortante resistente de cálculo $(\mathrm{kN})$;

$\mathbf{A}_{\mathbf{v}}=$ Área da seção transversal de aço resistente ao esforço cortante $\left(\mathrm{m}^{2}\right)$;

$\mathbf{f}_{\mathbf{y}}=$ Resistência ao escoamento do aço (kPa);

$\mathbf{V}_{\mathrm{a} 1}=$ Coeficiente de ponderação da resistência do aço, igual a 1,1 . 
Para perfis laminados $\mathrm{I}$ ou $\mathrm{H}$, com carregamento aplicado no plano da alma, $A_{v}$ não deve ser inferior ao produto da altura $\left(h_{w}\right)$ pela espessura $\left(t_{w}\right)$ da alma, e pode ser calculada conforme a Equação 5 .

$$
A_{v}=A-2 b t_{f}+\left(t_{w}+2 r\right) t_{f}
$$

\section{Em que:}

$\mathbf{A}_{\mathbf{v}}=$ Área da seção transversal de aço resistente ao esforço cortante $\left(\mathrm{m}^{2}\right)$;

A = Área da seção transversal sem a chapa adicional $\left(\mathrm{m}^{2}\right)$;

$\mathbf{b}=$ Largura da mesa do perfil $(\mathrm{m})$;

$\mathbf{t}_{\mathbf{f}}=$ Espessura da mesa do perfil $(\mathrm{m})$;

$\mathbf{t}_{\mathrm{w}}=$ Espessura da alma do perfil $(\mathrm{m})$;

$r=$ raio de concordância $(m)$.

A verificação da resistência à cortante é determinada de acordo com a Equação 6.

$$
\mathrm{V}_{\mathrm{Sd}} \leq \mathrm{V}_{\mathrm{Rd}}
$$

\subsubsection{Número de conectores de cisalhamento}

Para o dimensionamento do número de conectores de cisalhamento $\left(\mathrm{N}_{\mathrm{c}}\right)$ do tipo pino cabeça, considerando uma conexão total, primeiramente é preciso definir a força de cisalhamento $\left(F_{c}\right)$, isto é, a força necessária para garantir a transferência total do esforço cisalhante do concreto para o aço (BARROS, 2011).

Tendo ciência da posição da linha neutra plástica, deve-se determinar as forças resistentes do concreto, da chapa metálica adicional e da seção metálica restante, conforme definido nas Equações 7,8 e 9 , respectivamente.

$$
\begin{gathered}
R_{c}=\frac{0,85 f_{c k}}{\gamma_{c}} b_{e f f} y_{p} \\
R_{p}=\frac{f_{y}}{\gamma_{a 1}} A_{p} \\
R_{a}=\frac{f_{y}}{\gamma_{a 1}} A
\end{gathered}
$$

Em que:

$\mathbf{R}_{\mathbf{c}}=$ Força resistente do concreto $(\mathrm{kN})$;

$\mathbf{R}_{\mathbf{p}}=$ Força resistente da seção metálica restante $(\mathrm{kN})$;

$\mathbf{R}_{\mathrm{a}}=$ Força resistente da chapa metálica adicional $(\mathrm{kN})$;

$A_{p}=$ Área da chapa metálica adicional $\left(\mathrm{m}^{2}\right)$.

$\mathbf{A}=$ Área da seção transversal sem a chapa adicional $\left(m^{2}\right)$;

$\mathbf{f}_{\mathrm{ck}}=$ Resistência característica do concreto à compressão $(\mathrm{kPa})$;

$\mathbf{b}_{\text {eff }}=$ Largura efetiva da laje de concreto $(\mathrm{m})$;

Dessa maneira, conforme Barros (2011), entre uma seção de momento positivo máximo e outra de momento nulo, a força que deve ser transmitida na conexão é dada na Equação 10.

$$
F_{c}=\min \left(R_{c}, R_{a}+R_{p}\right)
$$

Finalmente, o número de conectores necessários à conexão total é calculado pela Equação 11.

$$
\mathrm{N}_{\mathrm{c}}=\frac{\mathrm{F}_{\mathrm{c}}}{\mathrm{Q}_{\mathrm{Rd}}}
$$

Em que:

$\mathbf{N}_{\mathbf{c}}=$ Número de conectores de cisalhamento;

$\mathbf{F}_{\mathrm{c}}=$ Força de cisalhamento $(\mathrm{kN})$;

$\mathbf{Q}_{\mathbf{R d}}=$ Força resistente de cálculo de um conector de cisalhamento tipo pino cabeça $(\mathrm{kN})$.

A norma EN 1994-1-1 (ECS, 2004) designa o cálculo de $Q_{R d}$ pelo menor dos dois valores apresentados nas Equações 12 e 13.

$$
\begin{gathered}
Q_{R d}=\frac{0,8 f_{u c s} A_{c s}}{\gamma_{c s}} \\
Q_{R d}=\frac{0,29 \alpha d^{2} \sqrt{f_{c k} E_{c}}}{\gamma_{c s}}
\end{gathered}
$$

Em que:

$\mathbf{f}_{\text {ucs }}=$ Resistência à ruptura do aço do conector (MPa);

$A_{c s}=$ Área da seção transversal do conector $\left(\mathrm{m}^{2}\right)$;

$V_{c s}=$ Coeficiente de ponderação da resistência do conector, igual a 1,25 para combinações últimas de ações normais;

$\mathbf{d}=$ Diâmetro da seção transversal do conector $(\mathrm{m})$; $\boldsymbol{\alpha}=$ Para $\left(\mathrm{h}_{\mathrm{cs}} / \mathrm{d}\right)>4$, igual a 1 ; Para $3 \leq\left(\mathrm{h}_{\mathrm{cs}} / \mathrm{d}\right) \leq 4$, igual a $0,2\left(\left(\mathrm{~h}_{\mathrm{cs}} / \mathrm{d}\right)+1\right)$;

$\mathbf{f}_{\mathrm{ck}}=$ Resistência característica do concreto à compressão $(\mathrm{kPa})$;

$\mathbf{E}_{\mathrm{c}}=$ Módulo de elasticidade do concreto $(\mathrm{kPa})$.

\subsection{FORMULAÇÕES NO ELS}

Devido à altura reduzida dos tipos de viga Slim Floor, deve-se dar atenção à verificação de serviço quanto à deformação excessiva (BARROS, 2011). 
O controle de flechas ganhou essa importância porque, dadas as características mecânicas dos materiais, tem sido possível aumentar o vão das vigas e diminuir a altura total do sistema misto. Por isso o controle de flechas é cada vez mais exigido, com o objetivo de prevenir fissurações nas seções e manter a estrutura adequada à condições frente aos critérios de durabilidade e estética (PAES 2003).

O cálculo das flechas depende basicamente da rigidez à flexão da viga em situação de serviço. Segundo Paes (2003), pode-se considerar o momento de inércia da seção mista não fissurada para estimar as flechas da viga utilizando o coeficiente de equivalência $\left(\alpha_{e}\right)$, e realizando os cálculos da deformação considerando as propriedades elásticas da seção.

As expressões para determinação da posição da linha neutra elástica $\left(\mathrm{y}_{\mathrm{e}}\right)$ e do momento de inércia $\left(I_{c}\right)$ da seção mista são dadas nas Equações 14 e 15, respectivamente.

$$
y_{e}=\frac{\frac{b_{\text {eff }}\left(D_{s}+D_{d}-h_{\text {eff }}\right)^{2}}{2 \alpha_{e}}+A_{a}\left(D_{s}+D_{d}-z_{1}\right)}{A_{a}+\frac{b_{\text {eff }}\left(D_{s}+D_{d}-h_{\text {eff }}\right)}{10}}
$$$$
I_{c}=b_{\text {eff }}\left(D_{s}+D_{d}-h_{\text {eff }}\right)\left(\frac{\left(D_{s}+D_{d}-h_{\text {eff }}\right)^{2}}{12}\right)+
$$$$
b_{\text {eff }}\left(D_{s}+D_{d}-h_{\text {eff }}\right)\left(\frac{\left(y_{e}-\frac{D_{s}+D_{d}-h_{\text {eff }}}{\alpha_{e}}\right)^{2}}{\alpha_{e}}\right)+
$$

$\mathrm{I}_{\mathrm{a}}+\mathrm{A}_{\mathrm{a}}\left(\mathrm{D}_{\mathrm{s}}+\mathrm{D}_{\mathrm{d}}-\mathrm{t}_{\mathrm{p}}-\mathrm{z}-1-\mathrm{y}_{\mathrm{e}}\right)^{2}$

\section{Em que:}

$\mathbf{y}_{\mathbf{e}}=$ Posição da linha neutra elástica $(\mathrm{m})$;

$I_{c}=$ Momento de inércia da seção mista $\left(\mathrm{m}^{4}\right)$;

$\boldsymbol{\alpha}_{\mathrm{e}}=$ Coeficiente de equivalência;

$\mathbf{D}_{\mathrm{s}}=$ Altura de concreto colocado in loco acima da chapa nervurada $(\mathrm{m})$;

$\mathbf{D}_{\mathbf{d}}=$ Altura da chapa nervurada $(\mathrm{m})$;

$\mathbf{b}_{\text {eff }}=$ Largura efetiva da espessura de concreto $(\mathrm{m})$;

$\mathbf{h}_{\text {eff }}=$ Altura total do perfil sem a chapa adicional (m);

$\mathbf{A}_{\mathrm{a}}=$ Área total de aço $\left(\mathrm{m}^{2}\right)$;

$\mathbf{t}_{\mathrm{p}}=$ Espessura da chapa adicional $(\mathrm{m})$;

$\mathbf{z}_{1}=$ Distância da linha neutra elástica até a superfície externa da mesa inferior $(\mathrm{m})$;
$\mathrm{I}_{\mathrm{a}}=$ Momento de Inércia em relação ao eixo y (abcissa) $\left(\mathrm{m}^{4}\right)$;

$\mathbf{z}=$ Altura total de seção de aço $(\mathrm{m})$.

Para uma viga sem escoramento temporário, a deformação devido às sobrecargas

$\left(\delta_{\mathrm{q}}\right)$ deve ser baseada nas propriedades da seção mista, utilizando um $\alpha_{\mathrm{e}}$ médio igual a 6,5 , visto que a sobrecarga é uma ação de curta duração. A deformação referente às cargas permanentes $\left(\delta_{p}\right)$, entretanto, deve ser baseada apenas nas propriedades da seção metálica (BARROS, 2011).

Assim, as deformações podem ser representadas pelas Equações 16 e 17 indicadas abaixo.

$$
\begin{gathered}
\delta_{\mathrm{q}}=\frac{5 \mathrm{qL}^{4}}{384(\mathrm{EI})_{\mathrm{c}}} \\
\delta_{\mathrm{p}}=\frac{5 \mathrm{pL}^{4}}{384(\mathrm{EI})_{\mathrm{m}}}
\end{gathered}
$$

Em que:

$\boldsymbol{\delta}_{\mathrm{q}}=$ Deformação devido às sobrecargas $(\mathrm{m})$;

$\delta_{p}=$ Deformação referente às cargas permanentes $(\mathrm{m})$;

$\mathbf{q}=$ Carregamento da etapa normal utilizando a combinação proposta pela NBR 8800 (ABNT, 2008) - $\left(\mathrm{CP} 2+\psi_{2}{ }^{*} \mathrm{SC} 2\right)(\mathrm{kN} / \mathrm{m})$;

$\mathbf{p}=$ Carregamento da etapa de construção utilizando apenas $\mathrm{CP} 1(\mathrm{kN} / \mathrm{m})$;

$\mathbf{L}=$ Comprimento da viga $(\mathrm{m})$;

$(\mathbf{E I})_{\mathbf{c}}=$ Rigidez da seção mista $\left(\mathrm{kN} \cdot \mathrm{m}^{2}\right)$;

$(\mathrm{EI})_{\mathrm{m}}=$ Rigidez da seção metálica $\left(\mathrm{kN} \cdot \mathrm{m}^{2}\right)$.

Segundo as recomendações da EN 1994-11 (ECS, 2004), o limite de deformação recomendado para $\delta_{q}$ é de $L / 360$. A flecha em relação à carga total não majorada $\left(\delta_{\text {tot }}=\delta_{q}+\delta_{p}\right)$ também deve obedecer a um limite, correspondente à L/200.

Geralmente, nas estruturas mistas de edifícios, as flechas devido às cargas permanentes representam a maior parte da deformação total. Quando a flecha total supera o valor limite permitido pela norma, pode-se realizar uma contra-flecha na viga, com o objetivo de reduzir os efeitos desestabilizantes das cargas.

\subsection{DADOS DO DIMENSIONAMENTO}

Com o intuito de gerar resultados comparativos entre a estrutura Slim floor e a 
estrutura mista de aço e concreto convencional, conhecida no Brasil por Steel Deck, foram criadas situações que envolvem as características dos materiais, o tipo de conector de cisalhamento e as ações atuantes na estrutura.

Antes disso, é preciso definir os tipos de viga e de laje para cada sistema. No caso do Slim Floor, os mesmos encontram-se descritos nos itens 4.1.2 e 4.1.3, respectivamente.

Para o sistema Steel Deck, foi utilizado um perfil tipo I duplamente simétrico, laminado, produzido pela Gerdau Açominas. A laje mista utilizada foi a MF-75, confeccionada pela Metform (2007), que possui nervuras com altura de $7,5 \mathrm{~cm}$ e o sistema completo apresenta altura total de $14 \mathrm{~cm}$. As propriedades da seção da chapa e o consumo de concreto devem ser extraídos do catálogo fornecido pelo fabricante, dispostos em seu sítio na internet.

As demais características serão descritas a seguir para ambos os sistemas.

\subsubsection{Materiais}

- $\quad$ Concreto estrutural: $\mathrm{f}_{\mathrm{ck}}=25 \mathrm{MPa} ; \mathrm{E}_{\mathrm{c}}=23,8 \mathrm{GPa}$;

- Aço para perfis laminados: ASTM A-572 Gr 50, $f_{y}=345 \mathrm{MPa}, f_{u}=450 \mathrm{MPa}, E_{a}=210 \mathrm{GPa}$;

- Peso específico concreto $=25 \mathrm{kN} / \mathrm{m}^{3}$;

- $\quad$ Peso específico aço $=78,5 \mathrm{kN} / \mathrm{m}^{3}$.

\subsubsection{Tipo de conector de cisalhamento}

O tipo de conector adotado para transmitir os esforços de cisalhamento da Slim Floor, foi o Stud Bolt Ciser 19 mm x $80 \_\mathrm{mm}$, com $32 \mathrm{~mm}$ de diâmetro de cabeça. Entretanto, o conector perde $5 \mathrm{~mm}$ ao ser soldado diretamente no Metal Base (viga). Assim, a altura final do conector é de $75 \mathrm{~mm}$.

Para a Steel Deck, foi necessário adotar o Stud Bolt Tru-Weld 19,1_mm x 127_mm, com $31,7 \mathrm{~mm}$ de diâmetro de cabeça para garantir a conexão total, foco do estudo. Neste caso, a conector perde 9_mm ao ser soldado através do Steel Deck. Assim, a altura final do conector é de $118 \mathrm{~mm}$.

O anel cerâmico, com configuração MB para Slim Floor e SD para Steel Deck, é colocado sobre o conector, delimitando a área de soldagem.

\subsubsection{Ações atuantes nas estruturas}

A Tabela 1 abaixo apresenta os dados da largura de influência da laje e das ações atuantes nos sistemas Slim Floor e Steel Deck.

TABELA 1: Vãos de laje e ações nas estruturas.

\begin{tabular}{ccc} 
& Slim Floor & Steel Deck \\
\hline Vão da laje $(\mathrm{m})$ & 6 & 3 \\
CP1 $(\mathrm{kN} / \mathrm{m})$ & Variável & 8,85 \\
CP2 $(\mathrm{kN} / \mathrm{m})$ & 13,3 & 9,94 \\
SC1 $(\mathrm{kN} / \mathrm{m})$ & 6 & 3 \\
SC2 $(\mathrm{kN} / \mathrm{m})$ & 9 & 4,5 \\
\hline
\end{tabular}

Legenda:

CP1 = Carga permanente antes da cura do concreto; CP2 = Acréscimo de carga permanente após a cura do concreto;

SC1 = Carga acidental antes da cura do concreto; SC2 = Carga acidental após a cura do concreto.

FONTE: Autoria própria.

O valor de CP1 não foi informado para o sistema Slim Floor, pois depende da altura total do sistema, ou seja, varia para cada perfil de viga utilizado.

Considerou-se larguras de influência das lajes distintas para os sistemas. Esse fato é devido à limitação do vão livre máximo para os dois tipos de forma de aço, sendo $6 \mathrm{~m}$ para o Slim Floor e $3 \mathrm{~m}$ para o Steel Deck.

\subsection{ESTUDO PARAMÉTRICO}

Foram realizados estudos paramétricos utilizando os perfis mais eficientes para variações dos comprimentos dos vãos das vigas. A partir daí, pôde-se avaliar a desenvoltura de cada sistema frente a parâmetros de peso do aço, altura total do sistema, consumo de concreto e deformação.

Para realizar o dimensionamento do Slim Floor foram desenvolvidas planilhas eletrônicas com a implementação da formulação no ELU e ELS.

O dimensionamento do Steel Deck foi realizado por meio do programa VigaMix.

\section{RESULTADOS E DISCUSSÕES}

Com o dimensionamento do sistema Slim Floor e através do programa VigaMix foi possível obter os parâmetros para construção dos gráficos (Figuras 5, 6, 7 e 8) dispostos a seguir. 


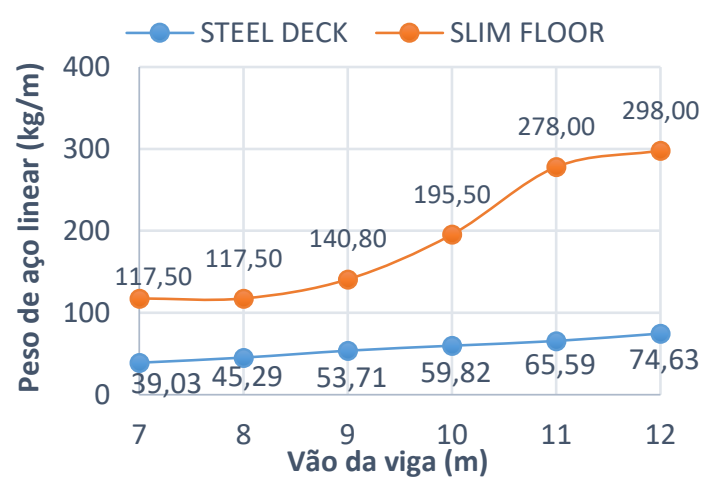

FIGURA 5: Peso de aço x Vão da viga FONTE: Autoria própria.

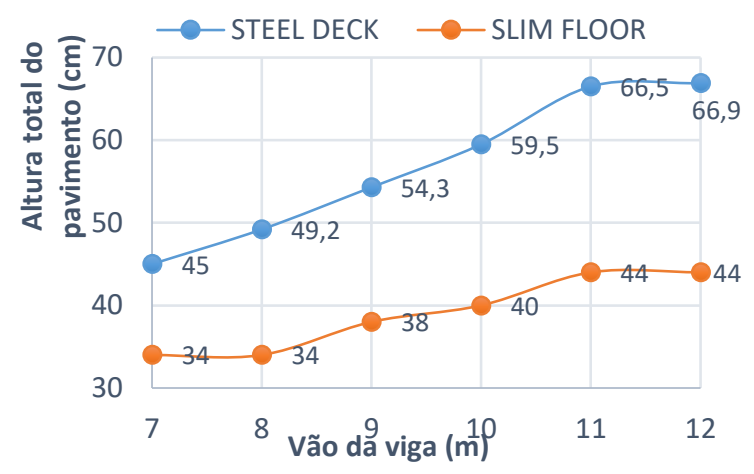

FIGURA 6: Altura do pavimento x Vão da viga FONTE: Autoria própria.

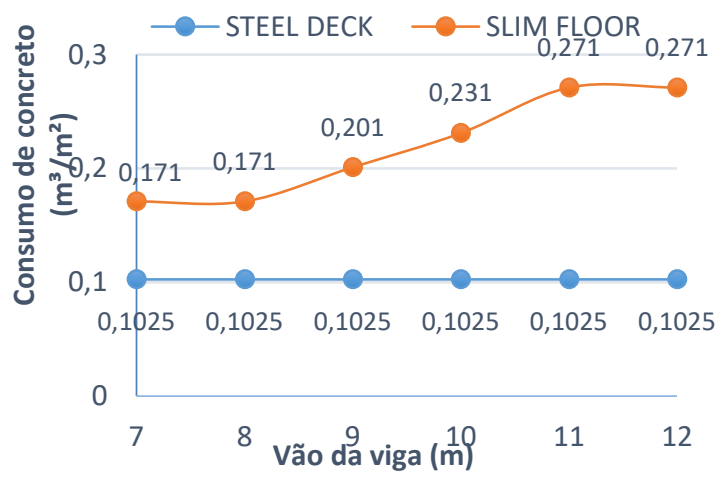

FIGURA 7: Consumo de concreto x Vão da viga FONTE: Autoria própria.

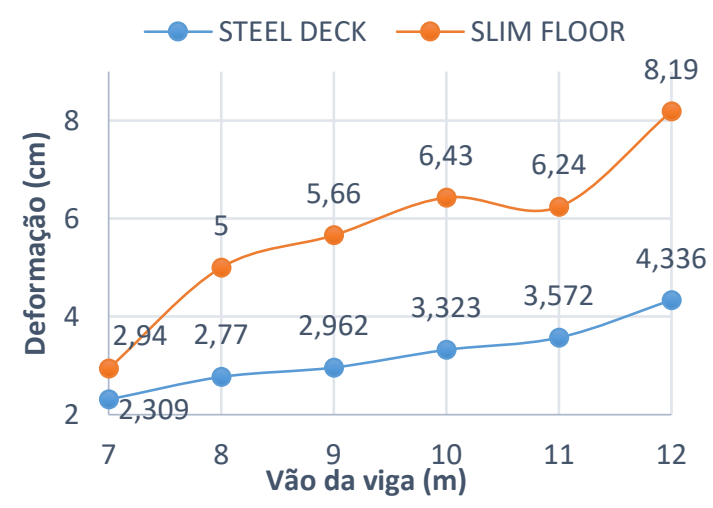

FIGURA 8: Deformação x Vão de laje FONTE: Autoria própria.
Com relação ao peso do aço (Figura 5), deve-se levar em consideração que um mesmo vão de viga no sistema Slim Floor atende a uma área de influência duas vezes maior do que no sistema Steel Deck. Isso implica que para cada viga de Slim floor, seriam necessárias duas vigas de Steel Deck, e consequentemente o peso do aço seria duplicado.

Contudo, verificou-se que o sistema Slim Floor se manteria mais pesado, apesar de diminuir consideravelmente a disparidade entre as estruturas. Mesmo com essa desvantagem, existem outras variáveis significativas que devem ser consideradas.

Quando, no Steel Deck, se utiliza o dobro de vigas, aumenta-se o tempo de execução, a quantidade de conectores os custos da mão de obra e da fabricação de uma quantidade maior de perfis. Existe, também, a necessidade de uma logística maior quanto ao deslocamento e armazenagem dos materiais.

No tocante à altura total dos sistemas (Figura 6), comprova-se a maior vantagem do Slim Floor. Há uma redução na altura do pavimento que varia de $11 \mathrm{~cm}$ a $22 \mathrm{~cm}$ em relação a Steel Deck. Assim, um edifício construído com Slim Floor apresentará uma altura total inferior à um edifício construído com Steel Deck, para um mesmo número de pavimentos. Além de reduzir o consumo de aço dos pilares, esta possibilidade pode ser útil em zonas onde existem restrições quanto à altura máxima dos edifícios.

O consumo de concreto (Figura 7) no sistema Steel Deck é constante porque a laje encontra-se sobre a mesa superior do perfil. Dessa forma, independente do perfil utilizado, não há necessidade de variar a altura da laje e, consequentemente, o consumo de concreto se mantém. Isso também implica que o carregamento devido ao peso próprio do concreto armado é constante, independente do vão da viga, contribuindo para uma variação das flechas inferior às vigas em Slim Floor, como explicitado na Figura 8.

Para a Slim Floor, quanto ao consumo de concreto, observa-se uma oscilação. Isso ocorre porque a laje encontra-se embutida na altura da viga. Assim, a quantidade de concreto empregada está diretamente relacionada à altura do pavimento 
que, por sua vez, baseia-se no perfil escolhido para cada situação criada ao modificar o vão da viga. Tal característica determina a variação do carregamento à medida em que se altera o perfil de aço.

A respeito da deformação o Slim Floor necessita de maiores cuidados, por se tratar de um piso de pequena altura. Como observado na Figura 8 , as flechas desse sistema são maiores que as do Steel Deck. Além da pequena altura e da influência do consumo de concreto, outro fator que contribui para a flecha elevada é o vão da laje, que é de $6 \mathrm{~m}$ para Slim Floor e de 3m para Steel Deck. Com um maior comprimento de vão, a área de influência da laje é maior, fazendo com que o carregamento ao longo da viga aumente, resultando em uma maior flecha.

Para ambos os sistemas empregou-se o artifício de contra-flecha, execução de uma curvatura inicial contrária àquela produzida pelas cargas solicitantes da estrutura, visando atender ao limite de deformação imposto pelas normas de aço. A flecha para o Steel Deck é limitada pela NBR 8800 (ABNT, 2008) e para o Slim Floor, pela EN 1994-1-1 (ECS, 2004).

A Tabela 2, a seguir, apresenta um resumo dos resultados do dimensionamento do Slim Floor obtidos através das planilhas eletrônicas.

TABELA 2: Resumo do Dimensionamento do Sistema Slim Floor

\begin{tabular}{|c|c|c|c|c|c|c|}
\hline & \multicolumn{6}{|c|}{ Vão (m) } \\
\hline & 7 & 8 & 9 & 10 & 11 & 12 \\
\hline Perfil & SFB HEB 240 & SFB HEB 240 & SFB HEB 280 & SFB HEB 300 & SFB HEB 300 & SFB HEB 300 \\
\hline Peso de aço $(\mathrm{kN} / \mathrm{m})$ & 117,50 & 117,50 & 140,80 & 195,50 & 278,00 & 298,00 \\
\hline Altura total $(\mathrm{cm})$ & 34,00 & 34,00 & 38,00 & 40,00 & 44,00 & 44,00 \\
\hline Concreto $\left(\mathrm{m}^{3} / \mathrm{m}^{2}\right)$ & 0,171 & 0,171 & 0,201 & 0,231 & 0,271 & 0,271 \\
\hline Deformação (cm) & 2,94 & 5,00 & 5,66 & 6,43 & 6,24 & 8,19 \\
\hline $\mathrm{M}_{\mathrm{pl}, \mathrm{Rd}}(\mathrm{kN} . \mathrm{m})$ & 840,58 & 861,38 & 1084,53 & 1702,72 & 2035,77 & 2312,65 \\
\hline$M_{\text {sd }}(k N . m)$ & 434,99 & 568,15 & 786,16 & 1058,89 & 1425,57 & 1701,83 \\
\hline$V_{R d}(k N)$ & 601,90 & 601,90 & 744,80 & 859,20 & 1639,70 & 1639,70 \\
\hline $\mathrm{V}_{s d}(\mathbf{k N})$ & 249,00 & 284,00 & 349,00 & 724,00 & 518,00 & 567,00 \\
\hline N. de Conectores & 47 & 52 & 59 & 70 & 84 & 93 \\
\hline
\end{tabular}

\section{CONSIDERAÇÕES FINAIS}

Ao desenvolver este trabalho tornaram-se mais evidentes algumas características do Slim Floor, sistema este que ainda é objeto de estudos.

Apesar de apresentar um peso maior, consumir mais concreto e possuir maiores flechas em relação a Steel Deck, a estrutura Slim Floor ainda é vantajosa ao considerar aspectos construtivos, econômicos, estéticos e, principalmente ao avaliar a grande redução de altura total do piso que este sistema proporciona.

Pôde-se observar o desempenho desse tipo de estrutura frente a vários parâmetros e então analisar sua eficiência em relação ao sistema misto convencional Steel Deck. Ao gerar os resultados apresentados, tornaram-se mais claros aspectos positivos e negativos desse sistema, oferecendo maiores condições para o desenvolvimento e utilização de novas possibilidades construtivas.

Para estudos futuros, o presente trabalho oferece uma orientação para o dimensionamento de Slim Flor nos ELU e ELS, visto que este sistema ainda não se encontra totalmente detalhado em normas. Dessa maneira, abre-se uma grande margem para novos testes com outros tipos de vigas e lajes específicas para o sistema, o que pode minimizar problemas e aprimorar qualidades identificadas nesta pesquisa.

\section{REFERÊNCIAS BIBLIOGRÁFICAS}

ASSOCIAÇÃO BRASILEIRA DE NORMAS TÉCNICAS, NBR 8800: Projeto de estruturas de aço e de estruturas mistas de aço e concreto de edifícios. Rio de Janeiro, 2008. 
ASSOCIAÇÃO BRASILEIRA DE NORMAS TÉCNICAS, NBR 6120: Cargas para o cálculo de estruturas de edificações. Rio de Janeiro, 1980.

BARROS, M. O. Análise e dimensionamento de pavimentos mistos slim floor. Tese (Mestrado em Engenharia Civil - Perfil de Estruturas) - Universidade Nova de Lisboa. Lisboa, 2011, 106 p.

EUROPEAN COMMITTEE FOR STANDARDIZATION. EN 1994-1-1: Eurocode 4 - Design of composite steeland concrete structures - Parte 1-1: General rules and rules for buildings. Bruxelas, 2004.

EN 1993-1-1: Eurocode 3 - Design of STEEL STRUCTURES - Part 1-1: General rules and rules for buildings. Bruxelas, 2010.

DE NARDIN, S; DE SOUZA, A. S. C.; EL DEBS, A. L. C. H.; EL DEBS, M. K. Estruturas mistas aço-concreto: origem, desenvolvimento e perspectivas. In: CONGRESSO BRASILEIRO DO CONCRETO, 47., 2005, Recife. Anais... Olinda: IBRACON, 2005, 68-84 p.

DE NARDIN, S. Pilares mistos preenchidos: estudo da flexo-compressão e de ligações viga-pilar. Tese (Doutorado) -, Universidade de São Paulo, Escola de Engenharia de São Carlos. São Carlos, 2003, 323 p.

DOS REIS, L. V. N. Lajes mistas com fôrma de aço incorporada: Aplicações, dimensionamento e metodologia de análise numérica. Trabalho de conclusão de curso - Universidade Federal de São Carlos. São Carlos, 2012.

GRIFFIS, L. G. The 1994 T.R. High lecture: Composite frame construction. In: NATIONAL STEEL CONSTRUCTION CONFERENCE, 5., 1994, Pittisburgh, 18-20 may, Proceedings... Nova lorque: AISC, 1994, 50-72 p.

MALITE; M. Análise do comportamento estrutural de vigas mistas aço-concreto constituídas por perfis de chapa dobrada. Tese (Doutorado) - Universidade de São Paulo, Escola de Engenharia de São Carlos, 1993.

METFORM, S. A. Manual Técnico: Especificações para Projeto, Manuseio e Montagem. Betim, 2007.

PAES, J. L. R. Aportaciones al análisis del comportamiento estructural de sistemas de forjados mixtos tipo "Slim floor". Tese (Doutorado em Engenharia da Construção) - Universidade Politécnica da Catalunha, Departamento de Engenharia da Construção. Barcelona, 2003, 385 p.

TATA STEEL, LTDA. Comflor composite floor deck brochure. Londres, 2013.

VIGAMIX, software versão 2.08: Vigas Mistas de Edifícios - Análise e Dimensionamento Paramétrico. Universidade Federal de Viçosa, 1998. 